\title{
How Old is Too Old? Breast Cancer Treatment in Octogenarians
}

\author{
Pedro Exman, MD and Harold J. Burstein, MD, PhD \\ Dana-Farber Cancer Institute, Brigham \& Women's Hospital, Harvard Medical School, Boston, MA
}

Breast cancer is largely a disease of older women. Of the 252,000 new breast cancers diagnosed in the United States in $2017,50 \%$ arose in women 65 years of age or older. ${ }^{1}$ These cancers are more likely to be ER positive, and less likely to be either triple-negative or HER2 positive, than cancers arising in younger women. Fortunately, breast cancer mortality has been steadily declining in the US for decades, the result of successful screening campaigns for early detection, and incremental improvements in adjuvant therapy. ${ }^{2}$ But not all age groups have seen similar reductions. Notably, the most profound improvements have been seen in younger women, while older women have seen fewer reductions in breast cancer mortality. Breast cancerspecific mortality rates declined between 1990 and 2007 by $2.49 \%$ per year $(95 \%$ CI $2.27-2.71 \%$ per year) in women aged between 20 and 49 years, $1.96 \%$ in women aged $65-74$ years and $1.14 \%$ in women aged 75 years and older. ${ }^{2}$ The causes for these discrepancies are not precisely known. In part, it relates to the historical underutilization of tamoxifen and ovarian suppression among young women with ER positive tumors, a situation now clarified in the literature. It also reflects marked improvements in antiHER2 therapy that have transformed outcomes for that breast cancer subtype, which is more common in younger women. Nonetheless, comorbidities, sub-optimal treatment and infrequent inclusion in clinical trials may be responsible for this deceleration of progress among elderly patients. The lower relative improvements in breast cancer mortality seen in older as opposed to younger women have prompted many to ask whether older women are being undertreated. In this current issue, Mamtani et al. present a

(C) Society of Surgical Oncology 2018

First Received: 12 February 2018;

Published Online: 9 April 2018

H. J. Burstein, MD, PhD

e-mail: hal_burstein@dfci.harvard.edu well-written portrait of the current real-life approach to early breast cancer in octogenarian patients.

First, this retrospective analysis demonstrated an overall breast cancer-specific mortality of only $5 \%$ in patients with stage 1 or II disease. Non-breast cancer conditions were 4 times more likely to be a cause of death in these women age 80 years and older. These findings are similar with previous populational studies that demonstrated that death due to breast cancer in older patients varies between 4.5 and $16.1 \%$ in stage I and II disease, respectively. ${ }^{3}$ The average life expectancy for an 80-year-old American woman is 10.1 years; it is 7.2 years for an 85 -year-old. Thus, it is essential to contextualize the risk of breast cancer versus other causes of mortality in older patients, and weight realistically the impact of breast cancer in patients with or without other major health concerns.

None of the patients in the report by Mamtani and colleagues went without surgical treatment for known breast cancer; however, 40\%-presumably all with a clinically negative axilla-received neither axillary surgery nor radiation therapy. This rate of omission of axillary dissection is similar to previous data from a large retrospective analysis which demonstrated that patients over 75 years of age were less likely to receive axillary dissection (84 vs. $93 \%, P=0.0003),{ }^{4}$ and seems like a well-crafted clinical decision-the rate of isolated local-regional recurrence was less than $2 \%$ through more than 5 years of follow-up. Radiation treatment was also used judiciously. The authors stratified patients into "high" or "low" risk based on grade (grade $3=$ high), HER2 status (positive = high) and ER expression (negative $=$ high). Among the high-risk cohort, $55 \%$ received radiation therapy, as did $36 \%$ of the low risk cohort. But 45 and 64\%, respectively, did not receive radiotherapy, and outcomes were still excellent. These results support prospective observations that radiation therapy can be safely omitted in treatment of older women with ER positive breast cancers receiving adjuvant endocrine therapy. ${ }^{5}$ 
Most notable in the report is the lack of chemotherapy utilization among octogenarians with early stage breast cancer. Not a single patient with low risk cancers (ER positive, grade 1 or 2 , and HER2 negative) received adjuvant chemotherapy. Of the "high risk" group, only $10 \%$ received chemotherapy, which was only done in women who had HER2 positive cancers, and also received anti-HER2 antibody treatment with trastuzumab. Said another way, in a large single institution experience of octogenarians being treated for breast cancer, no one received "adjuvant chemotherapy" aside from treatment for HER2+ cancers.

Prospective studies have shown that adjuvant chemotherapy is as effective in older women-usually defined as 65 or older-as in young women. But even in these canonical trials of treatment for elderly breast cancer patients, few of the study participants were over age 80. In CALGB 49907, a study that included women with stage IIII breast cancer, and which compared standard regimens (CMF or AC) against capecitabine, only 5\% of patients were greater than 79 years old. ${ }^{6}$ The ELDA trial, a trial specifically designed for patients with 65 years at diagnosis or older that compared taxane-based chemotherapy to CMF, excluded women older than age $79 .{ }^{7}$ In the randomized phase II trial ICE-II-GBG 52, a study developed specifically for women over 65 years with breast cancer, only $0.8 \%$ of patients were octogenarians. ${ }^{8}$ Collectively, these experiences suggest that the clinical community has drawn a line in the sand at age 80 when it comes to chemotherapy for stage I-II breast cancer.

One exception might be the combined use of chemotherapy with trastuzumab-based treatment in older women with HER2 positive breast cancer. Here, the therapeutic index seems more likely to favor chemotherapy treatment. The risk of recurrence is higher, and the therapeutic benefit more compelling. ${ }^{9}$ Yet there are data that older patients with HER2 positive tumors do not typically receive trastuzumab-based therapy. Freedman et al. used a SEER database analysis, to show that women over 80 were far less likely to receive standard treatment of trastuzumab (OR 15.3 95\% CI 9.92-23.67). ${ }^{10}$ Because of the epidemiology of HER2 positive breast cancer, there are rather few women who will be in their $80 \mathrm{~s}$ and receiving trastuzumab. But for such women, well tolerated chemotherapy regimens such as weekly paclitaxel in combination with trastuzumab may be appropriate. ${ }^{11}$

Mamtani and colleagues suggest that most patients tolerated treatment well, with few adverse events. This is reassuring, but the retrospective nature of this analysis, a selection bias of patients and the possibility that de-escalated regimens were utilized in this cohort means that the real rate of toxicity remains difficulty to know. Patients over 65 years of age undergoing chemotherapy are more likely to have grade 3 and 4 adverse events, higher risk of hospitalization and higher risk of cardiac events after Herceptin therapy when compared to younger patients. ${ }^{12,13}$ Because elderly women diagnosed with breast cancer are a heterogeneous population, initial functional assessment is mandatory when contemplating adjuvant treatment. ${ }^{14}$ More adequate than the ECOG performance status score, utilization of the Comprehensive Geriatric Assessment (CGA) has been shown to reduce early hospitalization and mortality in older patients with cancer by allowing clinicians to select who can and who cannot safely receive treatment. ${ }^{15}$ Others scores such as Cancer and Aging Research Group (CARG) and Chemotherapy Risk Assessment Scale for High Age (CRASH) help to predict the risk of toxicity with chemotherapy. ${ }^{15,16}$ Functional and geriatric assessment and life expectancy estimates in this patient population are more important than chronological age and should be the starting point for the decision of adjuvant treatment. That said, these projects included few of the oldest elderlywomen over 80 - and even those patients were highly selected based on considerations of general health and cognitive function.

It is important to ask whether the outcomes end-points used in conventional cancer studies are as relevant in the older population, given the greater risk of death from noncancer causes, and the powerful fear of loss of functionality. For many older patients, would the endpoint "dependent care free survival" be a more compelling measure of clinical benefit, or lack thereof? A recent metaanalysis showed that worsening of quality of life (QoL) was transient in elderly breast cancer patients treated with adjuvant chemotherapy, and that in the long term it does not affect patient's functionality. ${ }^{17}$ While this is a reassuring finding, the assembled cohort of "elderly" patients were largely between the ages of 65 and 80 , and thus may not truly be characteristic of treatment outcomes for the more senior and vulnerable spectrum of elderly.

By focusing on the outcomes for octogenarians, Mamtani and colleagues have broadened our understanding of what it means to treat older breast cancer patients. For those women with stage I or II breast cancer, judicious use of axillary surgery and radiation therapy, especially in $\mathrm{ER}+$ cases where patients can benefit from endocrine treatments, can yield excellent outcomes without overtreatment. Aside from management of HER2 positive tumors, adjuvant chemotherapy is relegated to a non-existent role in "average" risk early stage tumors. The studies of patients in their 80s underscore the important point that in clinic, there are the elderly, and then there are the really elderly. We need to know the difference. Clinicians should be aware of the many tools to help estimate survival and comorbidity when making treatment choices with older 
women, being careful not to impair hard-earned functionality and independence at this vulnerable time of life.

DISCLOSURES The authors have no financial and/or competing interests to disclose.

\section{REFERENCES}

1. SEER Stat fact sheet: Breast cancer National Cancer Institute http://seer.cancer.gov/statfacts/html/breast.html

2. Smith BD, Jiang J, McLaughlin SS, Hurria A, Smith GL, Giordano SH, Buchholz TA. Improvement in breast cancer outcomes over time: are older women missing out? J Clin Oncol. 2011;29:4647-53.

3. Schonberg MA, Marcantonio ER, Ngo L, et al. Causes of death and relative survival of older women after a breast cancer diagnosis. J Clin Oncol. 2011;29(12):1570-7

4. Owusu C, Lash TL, Silliman RA. Effect of undertreatment on the disparity in age-related breast cancer-specific survival among older women. Breast Cancer Res Treat. 2007;102:227-236.

5. Hughes KS, Schnaper LA, Bellon JR,et al. Lumpectomy plus tamoxifen with or without irradiation in women age 70 years or older with early breast cancer: long-term follow-up of CALGB 9343. J Clin Oncol. 2013;31(19):2382-7.

6. Muss HB, Berry DA, Cirrincione CT, et al. Adjuvant chemotherapy in older women with early-stage breast cancer. $N$ Engl J Med. 2009;360:2055-65.

7. Perrone F, Nuzzo F, Di Rella F, et al. Weekly docetaxel versus CMF as adjuvant chemotherapy for older women with early breast cancer: final results of the randomized phase III ELDA trial. Ann Oncol. 2015;26:675-82.

8. von Minckwitz G, Conrad B, Reimer T, et al. A randomized phase 2 study comparing EC or CMF versus nab-paclitaxel plus capecitabine as adjuvant chemotherapy for nonfrail elderly patients with moderate to high-risk early breast cancer (ICE IIGBG 52). Cancer. 2015;121(20):3639-48

9. Sun J, Chia S. Adjuvant chemotherapy and HER-2-directed therapy for early-stage breast cancer in the elderly. Br J Cancer. 2017;116:4-9.

10. Freedman RA, Vaz-Luis I, Barry WT, Lii H, Lin NU, Winer EP, Keating NL. Patterns of chemotherapy, toxicity, and short-term outcomes for older women receiving adjuvant trastuzumab-based therapy. Breast Cancer Res Treat. 2014;145:491-501.

11. Tolaney SM, Barry WT, Dang CT, et al. Adjuvant paclitaxel and trastuzumab for node-negative, HER2-positive breast cancer. $N$ Engl J Med. 2015;372(2):134-41.

12. Barcenas $\mathrm{CH}$, Niu J, Zhang N, et al. Risk of hospitalization according to chemotherapy regimen in early-stage breast cancer. J Clin Oncol. 2014;32:1-10.

13. Denegri A, Moccetti T, Moccetti M, Spallarossa P, Brunelli C, Ameri P. Cardiac toxicity of trastuzumab in elderly patients with breast cancer. J Geriatr Cardiol. 2016;13:355-63.

14. Biganzoli L, Wildiers H, Oakman C, et al. Management of elderly patients with breast cancer: updated recommendations of the International Society of Geriatric Oncology (SIOG) and European Society of Breast Cancer Specialists (EUSOMA). Lancet Oncol. 2012;13:e148-60

15. Hurria A, Togawa K, Mohile SG, et al. Predicting chemotherapy toxicity in older adults with cancer: a prospective multicenter study. J Clin Oncol. 2011;29:3457-65

16. Extermann M, Boler I, Reich RR, et al. Predicting the risk of chemotherapy toxicity in older patients: the chemotherapy risk assessment scale for high-age patients (CRASH) score. Cancer. 2012;118:3377-86

17. Cheng KKF, Lim EYT, Kanesvaran R. Quality of life of elderly patients with solid tumours undergoing adjuvant cancer therapy: a systematic review. BMJ Open. 2018;8(1):e018101 